\title{
Lumen
}

Selected Proceedings from the Canadian Society for Eighteenth-Century Studies

\section{Les Julie et Sophie de Charrière : Chassé-croisé avec Rousseau sur l'éducation des femmes}

\section{Marie-Hélène Chabut}

Volume 24, 2005

Indigenes and Exoticism

Indigènes et exotisme

URI : https://id.erudit.org/iderudit/1012179ar

DOI : https://doi.org/10.7202/1012179ar

Aller au sommaire du numéro

Éditeur(s)

Canadian Society for Eighteenth-Century Studies / Société canadienne d'étude du dix-huitième siècle

ISSN

1209-3696 (imprimé)

1927-8284 (numérique)

Découvrir la revue

Citer cet article

Chabut, M.-H. (2005). Les Julie et Sophie de Charrière : Chassé-croisé avec Rousseau sur l'éducation des femmes. Lumen, 24, 121-134.

https://doi.org/10.7202/1012179ar

Copyright (c) Canadian Society for Eighteenth-Century Studies / Sociéte canadienne d'étude du dix-huitième siècle, 2005
Ce document est protégé par la loi sur le droit d'auteur. L'utilisation des services d'Érudit (y compris la reproduction) est assujettie à sa politique d'utilisation que vous pouvez consulter en ligne.

https://apropos.erudit.org/fr/usagers/politique-dutilisation/ 


\section{Les Julie et Sophie de Charrière : Chassé-croisé avec Rousseau sur l'éducation des femmes}

"Quant à Rousseau et Voltaire, prenez-en votre parti : tous les saints de la légende seraient décanonisés, que ces nouveaux demi-dieux n'en réussiroient pas davantage. On peut dire du demi-dieu comme du grand homme, qu'il n'en est point pour son valet-de-chambre : or tous les lecteurs sont les valets-de-chambre de ces gens-ci ${ }^{1}{ }{ }$.

C'est en ces termes que Constance, dans Trois femmes (1798), affirme son refus de vouer un culte aux modèles en vogue, tout comme le fait Charrière dans sa correspondance post-révolutionnaire. Rousseau est présent très tôt dans la vie d'Isabelle de Charrière, et reste une forte présence et influence, toujours complexe et ambiguë, au fil de son œuvre, comme l'a montré Raymond Trousson ${ }^{2}$. À son excellente étude, qui insiste sur la distance prise peu à peu par Charrière d'avec le " rêveur " social et le pédagogue, sont venues s'ajouter des lectures qui prennent davantage en considération la question du gender et de la destinée féminine. Ainsi, pour Nadine Bérenguier, qui développe l'idée de «reading in pairs » de Nancy Miller ${ }^{3}$, les Lettres de Mistriss Henley constituent un commentaire sur l'échec du modèle domestique et raisonnable de

1 Isabelle de Charrière, Trois femmes et Suite dans CEuvres complètes, GA. Van Oorschot, vol. 9, Amsterdam/Genève, Slatkine, 1981, p. 109.

2 Raymond Trousson, "Isabelle de Charrière et Jean-Jacques Rousseau ", Bulletin de l'Académie Royale de langue et de littérature de Bruxelles, vol. 43, 1985, 5-57.

3 Nancy K. Miller, «Men's Reading, Women's Writing: Gender and the Rise of the Novel», Yale French Studies, 75, 1, 1988, p. 48-49: «Reading in pairs (...) By this I mean looking at the literature of men's and women's writing side by side to perceive at their points of intersection the differentiated lines of a "bi-cultural" production of the novel - Persian and Peruvian - more complicated than the familiar, national history of its tropes». 
Clarens ${ }^{4}$. D'autres critiques ont examiné la réception de Rousseau par ses contemporaines (Mme Roland, de Staël, de Genlis), parfois en observant la fonction dans une écriture féminine d'un prénom associé à Rousseau. C'est ainsi que Mary Trouille lit le texte de Mme D'Epinay intitulé Les Conversations d'Emilie (1773) comme une complexe réponse et un défi à la vision du rôle de la femme énoncée par Rousseau dans Julie et Emile ${ }^{5}$.

$C^{\prime}$ est dans cette perspective de réponse à ou de dialogue avec un discours modèle spécifique, celui de Rousseau sur le rôle et l'éducation des femmes, que j'examinerai l'usage qu'a fait Charrière de deux prénoms déjà fortement associés à Rousseau lorsqu'elle les choisit pour ses personnages féminins : Julie et Sophie. La fréquence de ces prénoms dans l'écriture de Charrière est frappante, en particulier dans son théâtre (Julie dans La Famille d'Ornac (1784), Monsieur d'Arget (1785), L'Emigré (1793) ; Sophie dans Comment la Nommera-t-on ? (1788) et L'Extravagant (1795)). Cependant, ils apparaissent également dans ses récits, et en particulier dans Le Noble (1762) et dans les fragments des Lettres de D'Arsillé fils, de Sophie Durfé et autres (1787-88), publiés pour la première fois dans les CEuvres complètes en 1981.

Devant l'ampleur de la tâche et la richesse de ces textes, je me limiterai ici aux deux courts récits mentionnés, mettant les pièces de théâtre de côté pour une lecture ultérieure. Mon propos, en «mettant en paire » ou en parallèle ces deux récits avec Julie et Emile, est de mettre en évidence un faire/jeu subversif de l'écriture de Charrière sur les idées de Rousseau et sur les deux représentations littéraires de la « femme idéale » qui naquirent sous sa plume de romancier et d'éducateur. Je montrerai comment ces prénoms, associés à la reprise quasi obsessionnelle et accélérée du scénario rousseauiste du destin féminin, constituent un arsenal de tactiques qui en amorcent une véritable réécriture. Je prends ici le terme tactique dans le sens où l'entendait Michel de Certeau dans L'Invention du quotidien lorsqu'il parlait des pratiques des consommateurs, c'est-à-dire des dominés, de ceux qui ne peuvent s'exprimer que de l'intérieur du lieu et du discours dominant, de l'autre ${ }^{6}$.

4 Nadine Bérenguier, "From Clarens to Hollow Park, Isabelle de Charrière's quiet revolution ", Studies in 18th Century Culture, 21, 1991, 219-43.

5 Mary Trouille, «La Femme Mal Mariée : Mme d'Epinay's Challenge to Julie and Emile », Eighteenth-Century Life, 20, 1, 1996, 42-66. L'auteur voit l'écriture féminine comme « an intertwining of dominant male and repressed female discourses » (p. 60).

6 Michel de Certeau, L'Invention du quotidien, 1980, p. 10-18. Au début de son texte sur les pratiques des consommateurs modernes, de Certeau distingue la stratégie, 
Cette lecture du Noble et des Lettres de d'Arsillé fils, de Sophie Durfé et autres s'attachera donc à mettre en évidence non seulement une mise en question d'un déjà écrit de la destinée féminine, mais une tentative de ré-écriture originale de cette destinée. L'intérêt du défi lancé dans ces deux textes par une voix féminine à celle d'un discours patriarcal est que Charrière, à la différence de la plupart de ses contemporaines, a choisi ici de reprendre point par point les données explicites des scénarios de Julie et d'Emile : appartenance et situation sociale, nom, âge, caractère des personnages, motifs de la lettre et de l'enlèvement, etc., tout en y introduisant une ou deux variables qui les font en quelque sorte s'effondrer. Cet essai a pour objet de montrer que c'est précisément en insinuant ces variables dans les données de la narration rousseauiste que Charrière parvient, en quelques pages, à inventer un récit qui redonne leur place, leur voix et leur bonheur aux personnages féminins figés et aliénés inventés par Rousseau.

\section{Julie d'Arnonville lectrice de Julie}

La jeune Belle de Zuylen écrivit en 1762 et publia en 1763, au grand dépit de sa famille et de ses proches, Le Noble, dont le sous-titre est conte moral. Ce court récit à la troisième personne plein d'ironie, peu étudié par les lecteurs de Charrière ${ }^{7}$, reprend le scénario classique des préjugés de classe paternels comme obstacle au mariage : Julie d'Arnonville aime Valaincourt, mais son père, obsédé par la grandeur ancienne de son nom, refuse catégoriquement l'union de sa fille avec un noble d'une seule génération. La Julie du Noble est ainsi mise, deux ans après la publication de La Nouvelle Héloïse, devant le même obstacle au bonheur que son homonyme. Valaincourt, comme Saint Preux, possède la noblesse du

pratique du "sujet de vouloir et pouvoir », de la tactique, « un calcul qui ne peut pas compter sur un propre, ni donc sur une frontière qui distingue l'autre comme une totalité visible. La tactique n'a pour lieu que celui de l'autre. Elle $s^{\prime} y$ insinue, fragmentairement, sans le saisir en son entier, sans pouvoir le tenir à distance " (p. 21-22). Jacqueline Letzter, dans son ouvrage sur Charrière, Intellectual Tacking, Amsterdam, Rodopi, 1998, a déjà utilisé le terme dans cette acception.

7 Starobinski le mentionne dans son article sur Les Lettres de Lausanne, Kathleen Jaeger y voit un roman féministe dans Male and Female Roles in the Eighteenth Century, Peter Lang, 1994, p. 139-160, et Jenene Allison, dans Revealing Difference: the Fiction of Isabelle de Charrière, U of Delaware Press, 1995, 21-27, l'associe aux Lettres trouvées dans des porte-feuilles d'émigrés et Sainte Anne et montre de manière convaincante la subversion d'une image stéréotypée de la femme qu'il opère. 
cœur sinon celle de ses ancêtres. Les pères des deux Julies ont un point en commun : rien ne les fera revenir sur leur décision : la loi paternelle ne peut être changée, elle est définitive et se prétend essentielle. Tout discours se révèle impuissant devant son autorité. Julie d'Etange est battue par son père qui provoque une fausse couche, bien que son acte et sa conséquence soient qualifiés à plusieurs reprises $\mathrm{d}^{\text {' } ~ " ~ a c c i d e n t ~ " ~ p a r ~}$ Julie. Quand au père de Julie d'Arnonville, il l'assaille de sa colère et la fait enfermer dans sa chambre. La loi du père est ainsi marquée, dans les deux textes, du sceau de la violence.

Et pourtant, du long roman épistolaire au court conte narré à la troisième personne, la destinée de Julie va changer de manière frappante. Alors que Julie d'Etange, malgré sa lucidité occasionnelle quant aux limites de sa destinée de femme ${ }^{8}$, ne peut se démarquer de celle-ci et accepte finalement tout de son père ${ }^{9}, \mathrm{y}$ compris un substitut de lui-même comme mari, Julie d'Arnonville, fille aimante mais railleuse, est posée d'emblée comme un personnage dangereux pour la loi et l'autorité paternelles :

Son père lui avait fait lire les traités du Blazon, qu'elle ne goutoit guère, et elle avoit lu quelques Romans qu'elle goutoit beaucoup plus [...] Un peintre qui copioit ses Grands-peres, \& leurs Quartiers, lui avoit donné des leçons de dessein; elle peignoit des paysages, $\mathcal{E}$ brodoit des fleurs ; [...] Elle étoit fort vive, \& fort gaie, quoique tendre, \& il lui échappoit quelquefois des railleries sur la noblesse, mais le

8 Julie a parfois le sentiment d'être l'esclave de son père, lorsqu'elle écrit à Claire après avoir reçu la lettre de Saint Preux qui lui propose de fuir avec lui. Elle s'exprime ainsi : «Enfin mon père m'a donc vendue ? Il fait de sa fille une marchandise, une esclave, il s'acquite à mes dépends ! il paye sa vie de la mienne ! » (p 94). Elle est parfaitement consciente du double standard qui détermine dans sa société l'éducation des hommes et des femmes, lorsqu'elle écrit à Saint Preux : "Considère dans nos communes infortunes l'état de mon Sexe et du tien, et juge qui de nous est le plus à plaindre? Dans la force des passions affecter d'être insensible ; en proye à mille peines paroitre joyeuse et contente ; avoir l'air serein et l'ame agitée ; dire toujours autrement qu'on ne pense ; déguiser tout ce qu'on sent ; être fausse par devoir, et mentir par modestie : voila l'état habituel de toute fille de mon âge. On passe ainsi ses beaux jours sous la tyrannie des bienséances (...) Mais on gêne en vain nos inclinations ; le cœur ne reçoit de lois que de lui-même ; il échappe à l'esclavage ; il se donne à son gré. Sous un joug de fer que le ciel n'impose pas on n'asservit qu'un corps sans âme " (p. 212).

9 Julie, après avoir été battue et frappée par son père, non seulement accepte sans discuter son verdict, mais écrit en ces termes à sa cousine Claire : «Je lui ai dit et je le pense, que je serois trop heureuse d'être battue tous les jours au même prix, et qu'il n'y a point de traitement si rude qu'une seule de ses caresses n'efface au fond de mon cœur. » (La Nouvelle Héloïse, CEuvres complètes, Paris, Gallimard, vol. 2, 1964, p. 176.) 
respect, et l'amitié qu'elle avoit pour son père, les modéroit toujours. Son père l'aimoit aussi, mais il auroit souhaité qu'au lieu de fleurs elle brodât sur les écrans des Armoiries ; qu'au lieu de Télémaque, $\mathcal{E}$ de Gilblas elle étudiât les parchemins rongés qui constataient les titres de la famille. ${ }^{10}$

Tout ce que son père fait apprendre à Julie, que ce soit la lecture, le dessin ou la peinture, est détourné par l'héroïne au profit de ses goûts personnels, avec joie, sans arrière pensée et sans regret. Et c'est ainsi que trouvent place dans ses lectures un roman picaresque aussi bien que le Télémaque de Fénelon qui sera le livre unique de Sophie dans Emile (mais tout roman, même pédagogique, n'est-il pas dangereux, comme nous le montre la fantaisie de l'autre Sophie, malheureuse d'idéal, dans $E_{\text {Emile }}^{11}$ ?). Alors que Julie d'Etange, en accord avec Saint Preux, le préfacier de la Nouvelle Héloïse et le narrateur-auteur d'Emile, condamne les " livres d'amour » ou "romans " ${ }^{12}$, à cause du danger qu'ils constituent pour la bonne éducation d'une jeune fille, la jeune fille éduquée et férue de romans qui écrit le Noble s'est empressée de mentionner les lectures préférées de sa Julie, clin d'œil évident au discours du maître. De plus, la gaieté de la Julie de Charrière contraste de manière frappante avec la notion racinienne de faute et d'aliénation associée au sentiment amoureux de la jeune Julie d'Etange pour qui l'amour est une véritable contamination, une maladie mortelle, pour reprendre les termes de Nancy Miller ${ }^{13}$.

Cet écart d'éducation et de perspective explique sans doute la réaction si différente des deux héroïnes découvrant l'amour. Alors que Julie d'Etange est écrasée par un sentiment de souffrance, de faute et de

10 Isabelle de Charrière, Le Noble, CEuvres complètes, GA, Van Oorschot, Vol. 8, Amsterdam/Genève, Slatkine, 1980, p. 21-22 (je souligne).

11 Rousseau, Emile, Oeuvres complètes, vol. 4, Paris, Gallimard, 1969, p. 760-63.

12 Dans la lettre XII de la première partie, Saint Preux envoie à Julie une « espèce de plan qui puisse réparer par la méthode le tort que les distractions ont fait au savoir » (Julie ou la Nouvelle Héloïse, CEuvres complètes, vol. 2, Paris, Gallimard, 1964, p. 57). Il décide de limiter ses lectures à des « livres de goût et de mœurs » (p.59) et ajoute : «J'ai laissé par égard pour votre inséparable cousine quelques livres de petite littérature que je n'aurais pas laissés pour vous. Hors le Pétrarque, le Tasse, le Metastase, et les maîtres du théâtre français, je n'y mêle ni poètes ni livres d'amour, contre l'ordinaire des lectures consacrées à votre sexe » (p. 61). A quoi Julie acquiesce en utilisant le mot de « romans » (p. 62).

13 «J'ai été élevée dans des maximes si sévères que l'amour le plus pur me paroissait le comble du déshonneur» (p. 50), écrit Julie à Saint Preux. Pour l'analyse de Nancy Miller, voir The Heroine's Text, New York, Columbia University Press, 1980, p. 97-98. 
déshonneur avant même l'acte sexuel, c'est le plaisir qui envahit l'être de la nouvelle Julie amoureuse: « Tant qu'elle avoit été avec Valaincourt, elle n'avoit songé qu'au plaisir de le voir, \& de l'entendre : quand elle ne le vit plus elle sentit la douleur d'en être séparée ; elle pensa aux moyens de le revoir, \& de le voir toujours " (p. 23).

Et dans les quelques pages qui constituent le conte de Belle de Zuylen, bien des éléments de l'histoire de la première Julie sont repris en accéléré. Ainsi, Valaincourt propose à Julie d'Arnonville de l'enlever, tout comme Saint Preux, et plus tard son ami Milord Edouard, l'ont proposé à Julie d'Etange. Les lettres des deux jeunes hommes se ressemblent fort : elles parlent de bonheur, de plaisir, et appellent la femme aimée dans les bras de son amant. Toutes deux affirment la supériorité des lois de l'amour sur celle du père. "C'est la première loi qu'il faut écouter » (92), écrit Saint Preux, et Valaincourt de reprendre comme en écho : « ses droits sont sacrés ». La loi du père devient dans sa lettre « le barbare préjugé » (31). Mais alors que Julie d'Etange refuse par deux fois de quitter sa famille, ou plutôt son père ${ }^{14}$, Julie $d^{\prime}$ Arnonville au contraire accepte la proposition qui lui est faite et agit pour la réaliser au plus vite. Charrière a pris soin d'émailler son texte de courtes phrases qui expriment l'hésitation ou le remords de l'hérö̈ne, mais qui sont très vite balayées par sa réflexion, sa volonté de choisir son destin et sa tension vers le bonheur.

14 A la lettre XXVI du premier livre, Saint Preux exhorte Julie à la fuite en ces termes : «Un éternel arrêt du ciel nous destina l'un pour l'autre; c'est la première loi qu'il faut écouter $[\ldots]$ tu veux forcer des barrières insurmontables et négliges les seuls moyens possibles [...] nous comptons déjà des années perdues pour le plaisir [...] O amante aveuglée! Tu cherches un chimérique bonheur pour un temps où nous ne serons plus ; $[. .$.$] reviens, il en est tems encore, reviens, ma Julie, de cette erreur funeste.$ Laisse là tes projets et sois heureuse. Viens, $\mathrm{O}$ mon âme, dans les bras de ton ami, réunir les deux moitiés de notre être : viens à la face du ciel guide de notre fuite et témoin de nos sermens jurer de vivre et mourir l'un à l'autre. Ce n'est pas toi, je le sais, qu'il faut rassurer contre la crainte de l'indigence. Soyons heureux et pauvres, ah quels trésors nous aurons acquis !» (p. 92-93). La lettre suivante est de Julie à Claire : «O si tu savois ce que l'insensé m'ose proposer !...et de quel ton !...m'enfuir ! le suivre! m'enlever !...Le malheureux ! »(p. 94), et elle pense à son père et à sa mère. Dans la seconde partie, Milord Edouard écrit à Julie pour lui proposer de venir en Angleterre vivre sur une de ses terres et de s'y marier avec Saint Preux (lettre III, p. 197-200). Il conclut ainsi sa lettre : « [...] craignez l'erreur des préjugés et la séduction des scrupules qui mènent souvent au vice par le chemin de l'honneur. [...] Je prévois ce qui vous arrivera si vous rejetez mes offres. [...] vous serez honorée et méprisable. Il vaut mieux être oubliée et vertueuse ". Dans la lettre VI, Julie refuse l'offre de M. Edouard en allégant ses devoirs envers son père et sa mère (p. 207-210). 
L'auteur du Noble joue ainsi sur le - et se joue du - terrible dilemme de la Nouvelle Héloïse ne pouvant choisir entre son père et son amant:

[...] si d'un côté son cœur se délectoit dans l'espoir d'être à son amant, de l'autre il saignoit pour son père. Ah ! que les principes d'une bonne éducation eussent été puissans sur une âme naturellement vertueuse, \& encore incertaine! Mais les arguments pour le devoir, qu'avoit toujours employés le Père, étoient encore moins solides que ceux de l'Amant pour l'amour (p. 32).

Le commentaire ironique du narrateur sur la différence qu'eût fait une «bonne éducation » projette dans le court récit l'image en filigrane de Julie d'Etange, mais pour mieux la dé-lire, puisqu'au devoir selon le père qui détermine la destinée de l'amante de Saint Preux se substitue bien vite, pour Julie d'Arnonville, le devoir amoureux : «(...) elle pensa être déterminée moins par sa passion que par un certain devoir inviolable que pourtant elle ne comprenoit pas » (p. 31).

De nouvelles valeurs, individuelles et nées de la sensibilité, se substituent à la rationalité sociale et morale, et Julie d'Arnonville écrit sa propre histoire, à la différence de Julie d'Etange qui doit y renoncer pour vivre dans le présent sans surprise et sans futur de Warens. Ce faisant, Le Noble réécrit, sur le mode euphorique, le texte dysphorique de $L a$ Nouvelle Héloïse ${ }^{15}$, et se termine sur ces mots : "Julie fut heureuse, \& ses fils ne furent point chevaliers » (p. 34).

Et, comble de l'ironie, Le Noble se joue du discours rousseauiste de $l^{\prime} E^{\prime} m i l e^{16}$ du fait même que c'est la bonté naturelle de Julie, jointe à un manque d'éducation " positive » — très proche de l'éducation négative $\mathrm{d}^{\prime}$ Emile, bien que sans raison pédagogique aucune et par le pur fruit du hasard - , qui lui permet de prendre ses distances d'avec le déjà écrit de sa destinée de femme ${ }^{17}$. Qu'en serait-il de Sophie, si le narrateur $\mathrm{d}^{\prime} E$ Emile

15 Je reprends ici la terminologie qu'utilise Nancy Miller dans The Heroine's Text, op. cit., pour classifier les romans anglais et français d'auteurs masculins dont les personnages principaux sont des femmes.

16 Emile en effet a déjà été publié (mai 1762) lorsque Belle de Zuylen rédige Le Noble en août 1762 .

17 «Il n'en faut pas beaucoup pour rendre polie une personne qui a l'esprit pénétrant et le cœur bon " (p. 21) et "Julie ne vouloit point avoir trop d'esprit, \& voilà pourquoi ce qu'elle en avoit plaisoit davantage. Elle savoit peu, mais on voyait que c'était faute d'occasion de pouvoir apprendre ; son ignorance n'avoit point l'air de la stupidité " (p. 22). 
lui avait donné l'éducation qu'il prône pour son jeune élève afin de laisser son esprit indépendant et libre de l'opinion $^{18}$ ?

\section{Sophie Durfé double inversé de Sophie}

Quelque vingt-cinq années plus tard d'ailleurs, en 1789, Charrière, dans son court pamphlet rousseauiste intitulé Courte réplique à l'auteur d'une longue réponse, un an avant d'écrire son assez inoffensif Eloge de Rousseau, affirmera sans ambiguité :

Je persiste à croire qu'une éducation qui donneroit de la force d'esprit aux femmes leur rendroit service ainsi qu'à la société.

J'ose faire à Rousseau un autre reproche. Il ne veut pas que les parents ou Instituteurs d'un jeune homme prennent sur eux de plier son caractère au joug de la société, d'après les idées qu'ils peuvent avoir de l'avantage qui en résulteroit pour lui, et il veut qu'on plie d'avance au joug la jeune femme. Or, les parents ont ce droit, ou ils ne l'ont pas. S'il leur est permis d'agir selon les convenances, telles qu'ils les conçoivent, ils le peuvent à l'égard du jeune homme comme de la jeune femme, dont les forces et les facultés, ou plutôt la faiblesse et les besoins, sont si semblables, à leur entrée dans la vie, qu'il n'est pas possible de croire qu'il y apportent des privilèges différents. [...] je laisserai mon fils et ma fille libres comme Emile, d'après les principes sur lesquels vous établissez sa liberté, ou, me croyant en droit d'user de mon expérience et de l'autorité que me donnent sur mes enfants ma tendresse et leurs besoins, je les accoutumerai tous deux, comme on y accoutumoit Sophie, avec adresse et douceur, aux entraves que ni l'un ni l'autre ne pourra toujours éviter ${ }^{19}$.

Charrière rejette ici le double standard, ou ce qu'elle nomme la « contradiction » qu'elle a observée dans les principes d'éducation exposés dans Emile. Mais nous allons voir qu'elle a déjà peu auparavant mis en fiction cette contradiction.

Le repérage des prénoms utilisés par Charrière m'a en effet amenée à la lecture des Lettres de D'Arsillé fils, de Sophie Durfé et autres ${ }^{20}$, texte quasi inconnu, inédit jusqu'à la publication des CEuvres Complètes et sans doute

18 Emile, p. 282, 303, 421.

19 Isabelle de Charrière, Courte réplique à l'auteur d'une longue réponse, CEuvres complètes, vol. 10, 1981, p.168-69.

20 Lettres de d'Arsillé fils, de Sophie Durfé et autres, CEuvres complètes, vol. 9, p. 657-78. 
le fruit d'une collaboration entre Charrière et Benjamin Constant vers 1787-88. Ce récit épistolaire inachevé nous donne la correspondance entre Sophie Durfé, roturière par son père mais riche, et son cousin d'Arsillé, qui joint à l'intelligence et à la beauté traditionnelles de l'amant un préjugé nobiliaire le rapprochant plutôt des personnages masculins du Noble. Sophie, qui aime son cousin, se voit confrontée à l'image des classes et de la destinée féminine que projette sur elle non plus son père mais l'homme qu'elle aime. Le père de Sophie, un des personnages masculins éminemment positifs de Charrière qui émailleront ses récits ultérieurs ${ }^{21}$, n'a de raison que le bonheur de sa fille (p. 670), et propose au père de d'Arsillé d'éloigner celui-ci de Sophie, qui est courtisée par sir George Lindsay, déterminé à l'épouser et à la rendre heureuse. Là s'interrompt le récit qui nous laisse pourtant un personnage féminin proto-féministe et, comme en préambule à la critique d'Emile que je viens de citer, une réflexion fascinante sur la place de la femme et ce qui « manque » à l'éducation de la Sophie de Rousseau.

Comme dans Le Noble, le préjugé de noblesse sert à Charrière de métaphore du scénario de la destinée féminine issu du discours patriarcal. Mais il est ici transmis par la mère de d'Arsillé et par le jeune homme $^{22}$. Ce récit pose ainsi la question primordiale de la responsabilité des femmes, et en particulier des mères, dans la transmission de ce que Simone de Beauvoir a appelé les «belles images » de l'idéologie dominante. Les rôles traditionnels du père et de l'amant qui met en danger sa loi sont ainsi inversés dans un scénario qui se complique : la loi traditionnelle du père devient ici indissociable de celle de l'amant. La phrase qui conclut la première lettre du roman, de d'Arsillé à Sophie, instaure d'emblée l'amant comme substitut du père et gardien des normes sociales. Recommandant en effet à Sophie de « renfermer » les livres qu'il a oubliés, il ajoute : "Ils vous conviendroient peu : les lectures d'une jeune fille doivent être choisies avec Soin. L'esprit à votre âge est ouvert à tant d'impressions ! et il faut beaucoup d'expériences pour éviter celles que de tels Livres peuvent produire ; à Dieu, ma belle » (p. 657). Con-

21 Je pense par exemple aux personnages de Laurent et Alphonse dans les Lettres trouvées dans des porte-feuilles d'émigrés (1793), de l'Abbé de la Tour, narrateur et personnage de Trois femmes et Honorine d'Userche (1798), ou de Sir Walter Finch dans Sir Walter Finch et son fils William (posthume, 1806, rédigé vers 1796).

22 Sophie juge ainsi d'Arsillé dans une de ses lettres: «vous répétez les paroles de ma tante (p. 660) ", et le père de Sophie reconnaît, dans sa lettre à son beau frère : "J'ai vu votre femme arracher du cœur de d'Arsillé le germe d'une passion honnête et douce $[\ldots]($ p. 669)». 
naissant la familiarité de Charrière avec Emile, il est difficile de ne pas penser ici au je éducateur de Rousseau. D'autant plus que Sophie Durfé rappelle étrangement, vue par la médiation du regard des autres, son homonyme. Elle est comme elle issue d'une mésalliance entre un homme riche et roturier et une femme noble ${ }^{23}$. Plus belle qu'elle, elle est comme elle douce et bonne ${ }^{24}$, elle a 15 ans $^{25}$ et elle a de l'esprit ${ }^{26}$ avec des connaissances très limitées.

Mais les ressemblances cessent dans le regard lucide de Sir George Lindsay qui voit ainsi Sophie : «Quant à l'esprit c'est le plus singulier mélange de la naïveté et de maturité. Quelque fois ses questions sont d'un enfant et d'autres fois sa pensée est profonde et son expression est forte et sentie » (p. 674-75). C'est que Sophie, ni enfant ni adulte, change. Et Sophie écrit, si elle ne lit pas. Elle s'inscrit alors peu à peu dans son écriture comme je en train de se faire. Dans Emile au contraire, Sophie ne vit que dans et par le discours du Je éducateur ou du Je amoureux. Comme la Julie de la petite société de Warens ${ }^{27}$, elle est en quelque sorte figée et fixée à jamais hors du temps dans l'essence immuable d'une image idéale de la féminité.

Sophie Durfé, elle, est saisie dans son devenir. Au fil de son existence et de son écriture, elle jette peu à peu au rebut l'autorité de celui que son rival le jeune Anglais qualifie de «tyran en graine » et de «Tarquin $»^{28}$. Ce rejet se fait $d$ 'abord par une mise en demeure qu'elle adresse au jeune d'Arsillé en ces termes : "Il faut que vous appreniez à respecter en moi la fille de mon père, \& si c'est trop de peine pour vous, je vous proteste que nous n'aurons plus ensemble que les conversations que je ne pourai éviter » (p. 660). Et ce faisant elle le défie de lui expliquer ce qu'est la

23 Cette information est donnée par le père de Sophie dans le " discours " que l'imagination du narrateur lui fait donner à la jeune fille : "Votre mère était de condition, j'étois riche ; voilà les seules considérations qui portèrent nos parents à nous unir. J'ai perdu mes biens, elle a perdu son nom » (p. 755).

24 Son père la décrit comme une jeune fille douce (p. 669).

25 Emile, p. 754.

26 Sa mère voit sa douceur et son esprit comme ses qualités les plus importantes (p. 666).

27 Pour une bonne étude sur la similarité de discours entre les deux textes de Rousseau, on lira l'article de R. J. Howells intitulé « Deux histoires, un discours : La Nouvelle Hélöise et le récit des amours d'Emile et Sophie dans l'Emile ", Studies on Voltaire and the Eighteenth Century, 249, 1987, p. 267-94.

28 "C'est la saison des Tulippes il faut que je me donne le plaisir de le mener dans un parterre, \& d'imiter le geste de Tarquin, Je parie qu'il m'entendra. Vrayment, c'est un tyran en herbe le plus décidé que j'aye jamais vu » (p. 674). 
noblesse et ajoute : « je respecterai ce que vous respectez, si je le puis ; si je ne le puis pas, nous n'en parlerons jamais». Bien loin de correspondre à l'image rousseauiste de la jeune femme modeste et dévouée au bonheur de l'homme qu'elle aime, elle affirme sur un ton autoritaire sa liberté de pensée et sa capacité de juger par elle-même ce que d'Arsillé considère comme une évidence indiscutable. Au fil de cette courte correspondance - six lettres sur les dix-huit qui constituent le fragment -, elle se fait plus sûre d'elle et assertive. Dans la lettre treize, elle en vient à usurper la prérogative " masculine " de la raison. Elle devient ainsi lectrice et critique de l'écriture de d'Arsillé qu'elle juge en ces termes : « Votre lettre est un Chaos Mon cher d'Arsillé \& on diroit que vous êtes un peu fou.[...]. Vos reproches ne sont pas raisonnables" (p. 667). Tout l'arsenal rhétorique d'un discours dominateur est concentré dans cette lettre : raisonnement sans faille, mise en évidence de contradictions ou de fausseté dans le discours de l'autre (p. 667), ironie même (p. 668).

Il est alors peu étonnant que cette lettre aboutisse à un démantèlement en forme du scénario de bonheur féminin conjugal et familial développé par le narrateur-éducateur d'Emile... et véhiculé plus maladroitement par d'Arsillé. Sophie Durfé en effet conclut ainsi sa longue lettre: « Une autre fois vous voulez contribuer à mon bonheur. Ce mot mon cousin a souvent une pauvre signification. Je connois une belle fille qui dit qu'elle contribue au bonheur de sa belle mere. Cela veut lui dire qu'elle met de la creme dans son cafe le matin, qu'elle consent à bailler tous les quinze jours à un souper de famille » (p. 668). Bien triste image au rabais de la félicité de la tendre épouse et mère que croit connaître Julie à Warens et que Sophie est en passe de connaître à la fin d'Emile sous le regard " éclairé » du précepteur/pseudo-père de son mari. On dirait ici que Charrière, comme si elle connaissait le sort qui attend la Sophie de Rousseau dans Emile et Sophie ou Les Solitaires, imagine ce que pourrait devenir l'existence de la femme d'Emile ${ }^{29} \ldots$

Après cette lettre, Sophie, faisant fi des convenances et des devoirs de la jeune fille selon Rousseau et sa Sophie ${ }^{30}$, écrit à d'Arsillé, dont elle a senti la tendance jalouse et tyrannique, pour lui demander de ne pas venir à une fête à laquelle elle se fait une joie de participer car, dit-elle,

29 Le texte inachevé d'Emile et Sophie ou Les solitaires, auquel Rousseau travaillait dès 1762, avait été publié par Moultou et du Peyrou dans la Collection complète des $\propto u v r e s$ de Jean-Jacques Rousseau (1782).

30 Emile, p. 751-52. 
« si vous en êtes vous montrerez de l'humeur (...), j'aurai sans cesse les yeux sur vous \& je ne serai ni gaye ni polie ni à mon aise » (p. 672). Charrière réécrit ici la scène du bal dans les Lettres de Mistriss Henley dans laquelle l'héroïne se sent mécontente d'elle-même, partagée, aliénée sous le regard de son mari. Sophie, encore jeune fille, essaie précisément d'échapper à ce regard qui l'objectivise. Et c'est bien parce qu'elle y réussit que le dernier dialogue - rapporté par le jeune Anglais - entre d'Arsillé et Sophie porte sur le changement de celle-ci. D'Arsillé, imbu de l'image fixée qu'il a de son rang et de la place que Sophie doit tenir dans son monde, lui déclare sur un ton de reproche : "Il faut [...] que je vous demande d'où vient le grand changement qui s'est fait chez vous en trois semaines. [...] Je vous ai laissé si bonne si douce [...] Mais de grâce répondez-moi. Qu'est-ce qui vous est arrivé pendant mon absence ? » (p. 677, je souligne). Malgré l'intervention du père qui exhorte le jeune homme à se régler « sur ce qu' elle est car » il ne saurait « la faire redevenir ce qu'elle étoit » (p. 677), il semble bien que Sophie ait pris des distances et des libertés avec sa destinée qui sont fort incompatibles avec une suite... de ses amours avec d'Arsillé.

Est-ce pourquoi le récit s'arrête quelques lignes après ces mots de Sophie : "Etant aussi une personne je trouve à propos d'avoir aussi une volonté » ? Du point de vue de la narration, l'absence de dénouement, trop caractéristique de l'écriture de Charrière pour être ignorée, comme l'ont montré Susan Jackson et Elizabeth MacArthur ${ }^{31}$, prend le contrepied de la clôture rassurante du livre $V$ d'Emile qui énonce/assure la permanence de la loi du père/précepteur sur la génération future. Tout, dans la dernière page du récit inachevé de Charrière, semble au contraire sonner le glas du scénario tant de fois répété, et avec tant d'art dans Emile, de la destinée féminine.

Charrière a créé avec Sophie Durfé un de ses personnages les plus forts et indépendants, en ce qu'elle n'a aucun des sentiments ambivalents d'aliénation d'une Mistriss Henley ou d'une Caliste. Cette force que son admirateur anglais voit en elle est associée à son milieu social, en particulier un père qui préfère « la sensibilité » (p. 654) à l'autorité, et à

31 Voir l'excellent article d'Elizabeth MacArthur sur les Lettres écrites de Lausanne de Charrière et les Lettres d'une Péruvienne de Graffigny, «Devious Narratives: Refusal of Closure in Two Eighteenth-Century Epistolary Novels», Eighteenth-Century Studies, $21,1,1987,1-20$. On lira également, pour une interprétation de la valeur novatrice et subversive du récit inachevé chez Charrière, l'article de Susan Jackson : «The Novels of Isabelle de Charrière, or, A Woman's Work is Never Done ", Studies in Eighteenth-Century Culture, 14, 1985, 299-306. 
son accès à l'écriture. " Je vois que vous me défendez presque de vous parler de M. de D. en suite vous me l'ordonnez. Sans me souvenir de l'ordre ni de la défense j'ai écrit ce qui s'est présenté à ma plume. » (p. 669, je souligne), écrit-elle à son amant. Ce qui est ordonné ou défendu, c'est-à-dire le devoir de la femme, coller à l'image qu'on lui a donnée à imiter, n'importe plus, quand la plume travaille. De là à faire de Sophie le négatif de la femme destinée à Emile, il n'y a qu'un pas. Après tout, que reste-t-il au futur mari, quand la femme s'est emparée de la faculté de raisonner, d'écrire et de décider ? Plus rien, dirait Rousseau, qu'une de ces femmes qui, comme Ninon de Lenclos (1620-1705) " se font hommes ". Et l'on se souvient ici de la longue diatribe du narrateur $\mathrm{d}^{\prime} E$ mile contre les femmes de lettres ${ }^{32}$ qui a marqué la jeune Belle de Zuylen au point qu'elle en a paraphrasé les dernières lignes dans un poème écrit en 1764 à James Boswell qui lui proposait d'envoyer certains de ses textes à Jean-Jacques:

[...] Un peu de vers et de Philosophie

Avec Rousseau me brouilleroit

A tout venant il crierait

Non, ce n'est pas là ma Sophie

Fille à brochures et Billets,

Qui ne fit onc manchettes ni lassets.

Ni Savonnage ni Purée

Mais des contes et des portraits

En un mot Fille un peu lettrée

Doit rester Fille à jamais ${ }^{33}$.

Ainsi, à deux moments de sa vie fort éloignés dans l'espace et dans le temps, Isabelle de Charrière, assumant son rôle de valet-de-chambre des grands hommes, commence et continue sa révision féministe de l'image littéraire de la femme et de son destin que lui ont transmis les lectures qui l'ont nourrie. Et les deux courts récits Le Noble et les Lettres de d'Arsillé fils, de Sophie Durfé et autres deviennent, par le biais des prénoms qu'ils empruntent à Rousseau et du jeu qu'ils opèrent sur les œuvres modèles, de véritables suppléments à l'écriture du maître qui visent à combler un manque qu'ils y ont décelé. Le dictionnaire Littré définit en effet ainsi le

32 Emile, p. 768. "Toute fille lettrée restera fille toute sa vie, quand il n'y aura que des hommes sensés sur la terre» .

33 Isabelle de Charrière, CEuvres complètes, vol 10, p. 345. 
supplément d'un livre : «ce qui est ajouté à un livre pour suppléer ce qui manquait ». Statut secondaire dira-t-on, que celui-ci ; mais tout supplément ne porte-t-il pas en lui le potentiel d'invalider le discours sur lequel il se greffe et de s'y substituer?

L'on peut toujours rêver...

MARIE-HÉLÈNE CHABUT

Lehigh University 